\title{
Management of Highly Placed Canine with Multi-Loop
}

\author{
Dr Dennis Lim,' Dr Pushkar Manandhar, 2 Dr Dharma Laxmi Basukala³ \\ 'Professor, ${ }^{2.3 R e s i d e n t, ~ D e p t ~ o f ~ O r t h o d o n t i c s ~}$ \\ University of the East, Manila, Philippines
}

Correspondence: dr.pushkar.manandhar@gmail.com

\section{ABSTRACT}

Maxillary canines are known as the corner stones of mouth. They are considered to be important for esthetics and for functional occlusion. Any disturbance in the eruption process leading to an aberrant position will hamper esthetics as well as function. Such conditions can be treated orthodontically in various ways including the alignment of canine into proper arch. This report describes two cases of leveling and alignment of highly placed canine into proper occlusion using multi-loop.

Key words: ectopic eruption, highly placed canine, multi-loop

\section{INTRODUCTION}

The prevalence of ectopic eruption or impaction of canine is $1-2 \%$ of the general population; ${ }^{1,2}$ in which palatally displaced canine is twice as much of the buccally placed canine.1.2,3 Ironically, buccally placed canines are seen more in clinical practice.

The causes for ectopic eruption or impaction of canine can be due to systemic and local factors. Environmental factors may contribute during the long tortuous eruption path of a canine. Specific etiologies include lack of space, early loss of a primary canine, ankylosis, root dilacerations, and an abnormal lateral root position in relation to an erupting canine. ${ }^{4}$ Another possible explanation is the disturbance associated with the follicle of unerupted tooth, that influence the direction of eruption and displacement of canine. ${ }^{5}$ Since the normal eruption path of the permanent canine is slightly buccal to the line of arch, reduced space in the canine area together with the close proximity of the adjacent teeth will prevent the canine from taking up its normal position in the arch and it will remain buccally displaced. ${ }^{6}$

\section{Case report 1}

An 18-years-old girl presented with skeletal class I and ectopically erupted maxillary canines on both sides with transposition of canine on right side (Figure 1A). The treatment objective were to bring canines to its proper alignment and position, and to achieve Class I canine relation. The treatment plan was extraction of maxillary first premolars and bring canine to proper level and alignment with multiloop. During leveling and alignment stage, 0.016 stainless steel arch wire with boot loop mesially and small omega loop were placed distally (Figure 1B). In almost three months canines were aligned and leveled. Presently the case is in the anterior retraction stage.
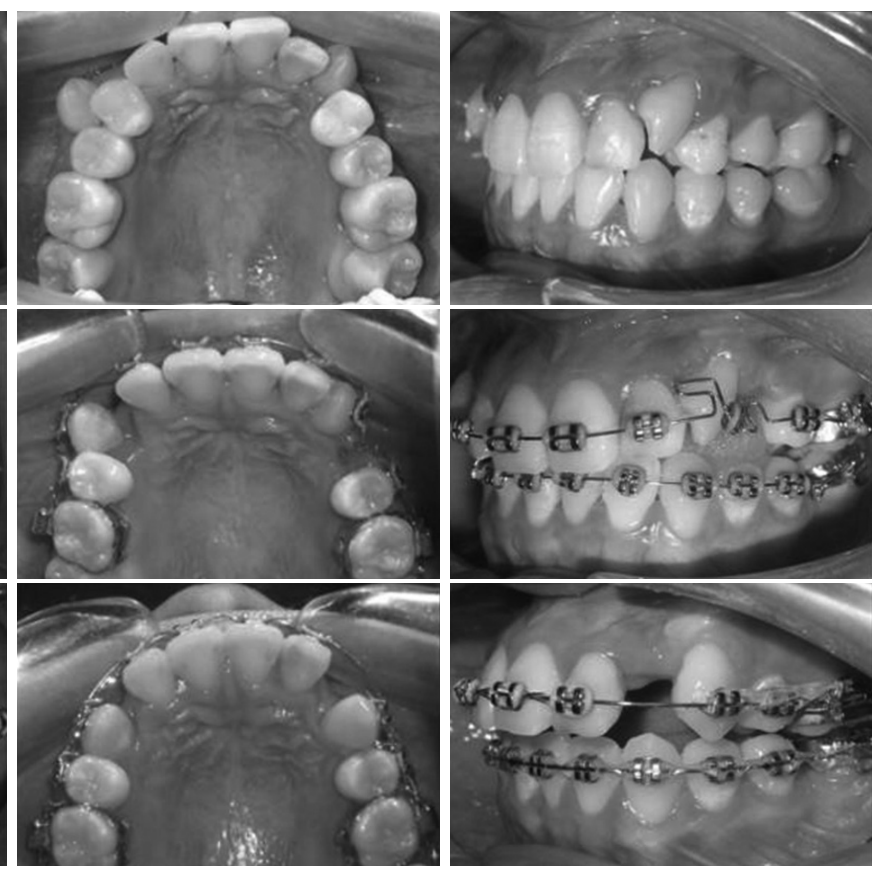
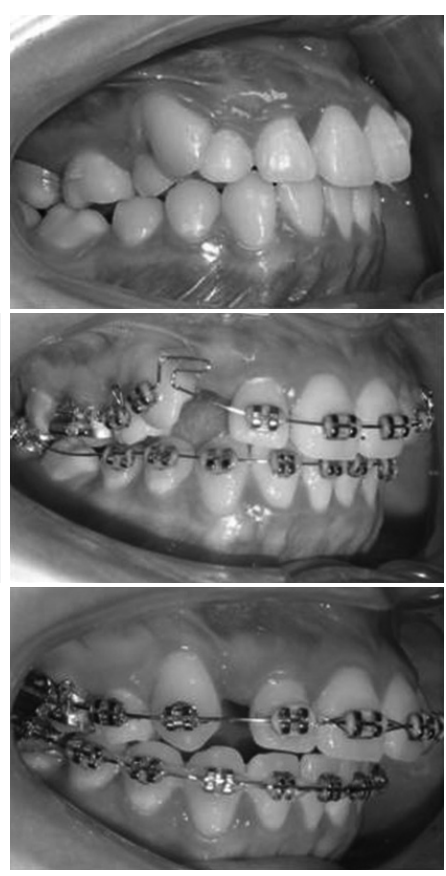

Figure 1: A. Pre treatment, B. With the multi loops, C. After the retrieval of canine in occlusion 


\section{Case report 2}

A 17-years-old girl came with a chief complain of unesthetic smile due to malaligned canine. Upon diagnosis she was found to be skeletal Class I with Class II canine and proclined upper anterior teeth (Figure 2A). The canine on the left side was highly placed and rotated. The treatment plan was to extract upper second premolars due to carious lesion. To level and align the upper left canine, a box loop was placed (Figure 2B). Within four months, the rotated highly placed canine was properly leveled and aligned. Presently, the case is in canine retraction phase.

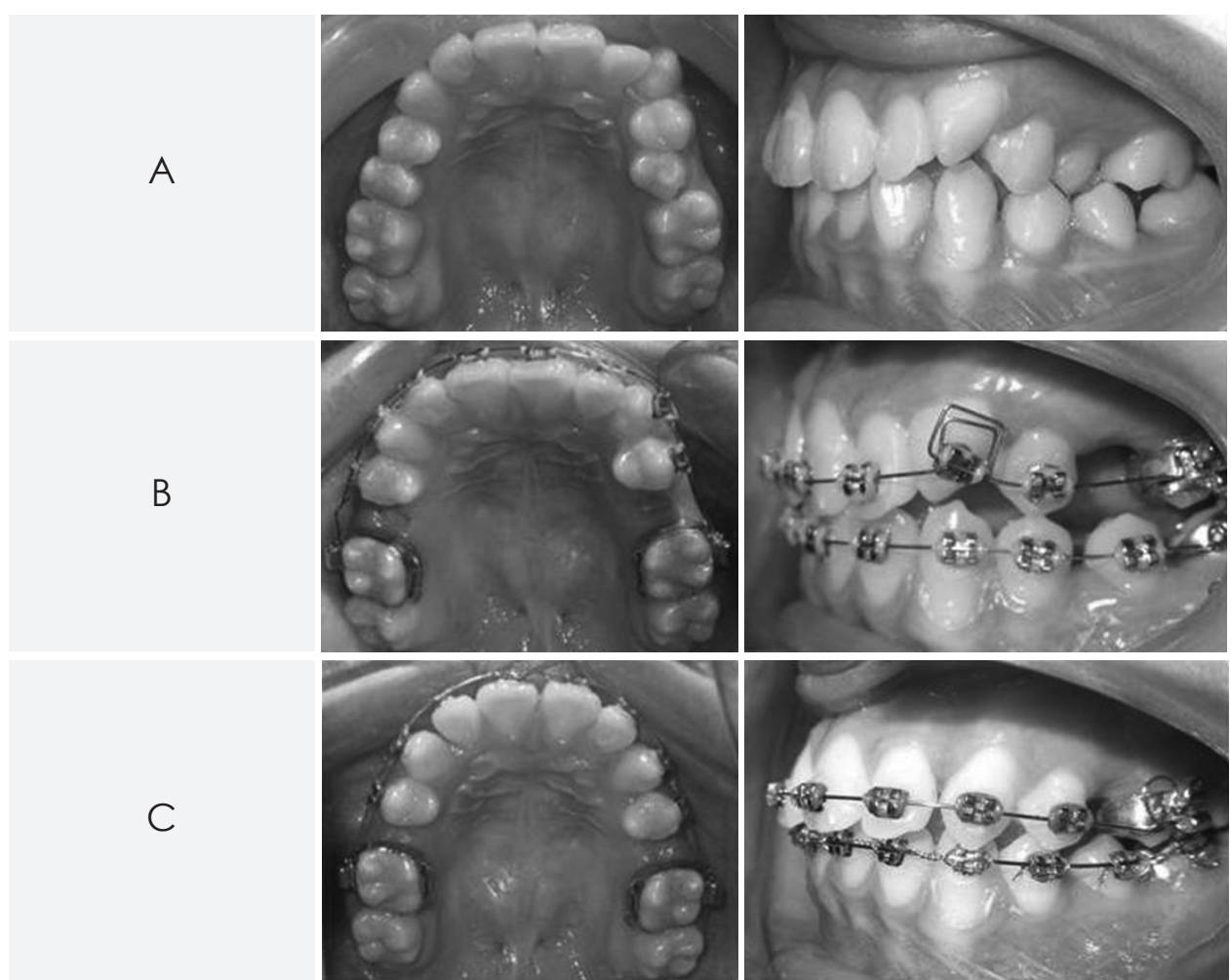

Figure 2: A. Pre treatment, B. With the multi loop (box loop), C. After the retrieval of canine in occlusion

\section{DISCUSSION}

In orthodontic practice, it is common to have patients with ectopic eruption of canine, in which buccally erupted canines are mostly encountered. Most of the cases are treated with NiTi flexible wire engaged in rotated and highly placed canine during the leveling and alignment stage. Flexibility of the NiTi wire pulls the canine in proper level and alignment with the anchorage from the adjacent tooth; in which lateral incisors and premolars are usually the anchor teeth. However during the process of alignment of buccally placed canine, there is some intrusion of the anchorage unit satisfying the Newton's third law of motion. Finally leaving with a distorted occlusal plane.
In contrary, the retrieval of rotated and highly placed canine with multi-loop in a stainless steel wire resulted to a minimal chance of distortion of the ooclusal plane. The multi-loop creates a break in continuity of arch wire resulting in minimal side effect to the anchorage unit. The stainless steel multi-loop wire provides flexibility without compromising the strength of the wire providing stability to the anchorage unit. ${ }^{7}$ As a result, there is minimal distortion to the occlusal plane.

The multi-loop (e.g. box loop, L-loop, T-loop etc) has horizontal and vertical bends which bring rotated highly placed canine to its position with less treatment time and minimal side effect to the anchorage unit.

\section{REFERENCES}

1. Bedoya MM, Park JH. A review of diagnosis and management of impacted maxillary canines. J Am Dent Assoc 2009;140:1485-93.

2. Fleming P, Scott P, Heidari N, Dibiase A. Influence of radiographic position of ectopic canines on the duration of orthodontic treatment. Angle Orthod 2009;79:442-6.

3. Cooke J, Wang HL. Canine impactions: Incidence and management. Int J Periodontics Restorative Dent 2006;26:483-91.

4. Jacoby H. The etiology of maxillary canine impaction. Am J Orthod. 1982;84:125-89.

5. Fearne J, Lee RT. Favorable spontaneous eruption of severely displaced maxillary canines with associated follicular disturbance. Br J Orthod. 1988; 115:93-8.

6. Becker A. The orthodontic treatment of impacted teeth. London Martin Dunitz Publishers; 1998.

7. Proffit WR, Fields HW, Sarver DM: Contemporary Orthodontics. 4th ed, Mosby Elsevier, 2000. 